\title{
Disminución en el tiempo de enfriamiento en la producción de achiote en barra
}

\section{Decreased cooling time in annatto bar production}

\author{
Mitwel Díazl, Christ Henríquez, Lissa Vegal, Katherina Vidal ${ }^{l}$ Rita Araúz $z^{1 *}$ \\ Licenciatura en Ingeniería Mecánica. Facultad de Ingeniara Industrial, Universidad Tecnológica de Panamá
}

\begin{abstract}
Resumen En este estudio se presenta el análisis de la variable tiempo de enfriamiento de las bandejas de achiote, escogida por ser una de las variables que más afecta el proceso de fabricación, el cual será analizado en base a los estándares de calidad cuidando cada detalle que se puede presentar en dicho proceso. Los datos son estudiados por medio de gráficos de control estadístico, y Seis Sigma. Los resultados obtenidos luego de haberse implementado las dos mejoras demuestran que se consiguió lograr el control del proceso.
\end{abstract}

Palabras clave Tiempo de enfriamiento, calidad, Seis Sigma, achiote, gráficos de control estadístico.

\begin{abstract}
This study presents the analysis of the variable cooling time of annatto trays, chosen for being one of the variables that most affects the manufacturing process, which will be analyzed based on quality standards, taking care of every detail in the process. The data are studied by means of statistical control charts, and Six Sigma. The results obtained after the two improvements have been implemented demonstrate that control of the process was achieved.
\end{abstract}

Keywords Cooling time, quality, Six Sigma, annatto, statistical control charts.

*Corresponding author: rita.arauz@utp.ac.pa

\section{Introducción}

El propósito de esta investigación es el mejoramiento del proceso de elaboración del producto del achiote condimentado en una empresa de la localidad. Este proceso de fabricación es de forma manual, por lo tanto, es un proceso largo, por la cantidad de tiempo que se debe esperar a que las bandejas lleguen a una temperatura ambiente que permita la solidificación de la mezcla.

Este artículo tiene como objetivo comparar los datos recolectados antes y después de las mejoras propuestas y poder determinar los factores que afectan el tiempo de enfriamiento de las bandejas.

Se considera que logrando disminuir este tiempo de enfriamiento, se podrá aumentar la producción y la productividad laboral de este producto; y aquí radica la importancia de esta investigación, dada la alta demanda de este producto en el mercado panameño. El achiote es un colorante natural utilizado en distintas gastronomías principalmente las latinoamericanas y orientales.

\section{Revisión literaria}

El achiote o como pocos lo conocen Bixa orellana [1] es una especie botánica absorbente de las regiones de América. El achiote en barra consiste en una mezcla de achiote con grasa vegetal, harina y componente. De su fruto se obtiene un colorante natural utilizado para diversos propósitos. En este caso, hablamos comercialmente, se utiliza para fines de coloración o condimento a las comidas. Se puede decir que, en la actualidad, hay muy pocas empresas que se dediquen a la producción de este tipo de especias.

El estudio se realizó en una empresa de la localidad, donde se desea implementar algunas mejoras que ayuden a tener una producción óptima, en cuanto al tiempo de secado de la mezcla de achiote antes de ser empacada y distribuida, cuidando ciertos estándares de la calidad. Otras variables a estudiar son el tiempo final de cocción, la cantidad de merma y la posición en que se coloquen las bandejas en los estantes para enfriarse. La gestión de la calidad es el procedimiento y técnica de planear, organizar, dirigir y controlar el uso de recursos para poder lograr cumplir ciertos objetivos tanto, específicos como generales, obteniendo mejoras continuas en cuanto a calidad y producción. Deming implementó el uso de la estadística dentro de los procesos productivos, así como la filosofía ahora conocida como Ciclo de Mejora Continua o Ciclo Deming que consiste en Planear - Hacer - Verificar y Actuar [2]. De acuerdo a lo anterior, se destacan las siguientes definiciones:

- Tiempo de enfriamiento: tiempo que demora la mezcla en tomar forma y compactarse dentro de los moldes.

- Tiempo de cocción: temperatura a la que se extrae el producto de las pailas.

- Merma: producto restante que se extrae de las bandejas, disminuye la temperatura de la mezcla y se puede lograr un tiempo de enfriamiento menor. 
- Capacidad: se le conoce como la amplitud de la variación natural del proceso para una característica de calidad dada [3].

- Seis sigmas: concepto que plantea una aspiración o meta común en calidad para todos los procesos de una organización [4].

- Control estadístico: disminuye variabilidad de procesos y mejora la calidad, disminuye desperdicios. Reúne muchas aplicaciones de herramientas matemáticas como diagramas de Pareto, índices de capacidad, control charts, Seis Sigmas, mediciones de productividad [2].

\subsection{Hipótesis}

Los estudios realizados dan como resultado dos hipótesis:

- Una vez se alcance el tiempo de enfriamiento deseado, se verán cambios en cuanto a producción y consistencia del producto. A menor tiempo de enfriamiento, se obtendrá mayor eficiencia de producción.

- Con la aplicación de la merma del producto en la mezcla caliente $\left(78^{\circ} \mathrm{C}\right)$, se obtiene una disminución en la temperatura de la mezcla. Temperatura propuesta de $45^{\circ} \mathrm{C}$ $50^{\circ} \mathrm{C}$.

\section{Metodología}

\subsection{Datos recolectados antes de mejoras}

La recolección de los datos se basó en un subgrupo (SG) de diez mediciones que se realizaron en distintos días, en donde se medía el tiempo de enfriamiento en minutos de las siete bandejas obtenidas de una paila de achiote condimentado. En la tabla 1 a continuación se presentan los datos obtenidos. Con estos datos se realizan los gráficos de control estadístico que se muestran en las figuras 1 y 2 , en donde se observa que el proceso no se encuentra en control estadístico.

Tabla 1. Datos recolectados del tiempo de enfriamiento, en minutos, de las bandejas de achiote condimentado en minutos

\begin{tabular}{|c|c|c|c|r|r|r|r|}
\hline $\mathbf{9 G}$ & 1 & 2 & 3 & 4 & 5 & 6 & 7 \\
\hline $\mathbf{1}$ & 108 & 89 & 105 & 87 & 85 & 84 & 83 \\
\hline $\mathbf{2}$ & 115 & 95 & 120 & 105 & 92 & 98 & 91 \\
\hline $\mathbf{3}$ & 86 & 104 & 85 & 86 & 87 & 77 & 65 \\
\hline $\mathbf{4}$ & 144 & 133 & 141 & 117 & 79 & 66 & 112 \\
\hline $\mathbf{5}$ & 100 & 76 & 92 & 64 & 72 & 73 & 78 \\
\hline $\mathbf{6}$ & 77 & 73 & 77 & 74 & 80 & 75 & 77 \\
\hline $\mathbf{7}$ & 74 & 70 & 76 & 74 & 63 & 75 & 75 \\
\hline $\mathbf{8}$ & 100 & 96 & 89 & 78 & 86 & 80 & 75 \\
\hline $\mathbf{9}$ & 107 & 98 & 84 & 86 & 75 & 65 & 79 \\
\hline $\mathbf{1 0}$ & 99 & 70 & 89 & 69 & 79 & 87 & 90 \\
\hline
\end{tabular}

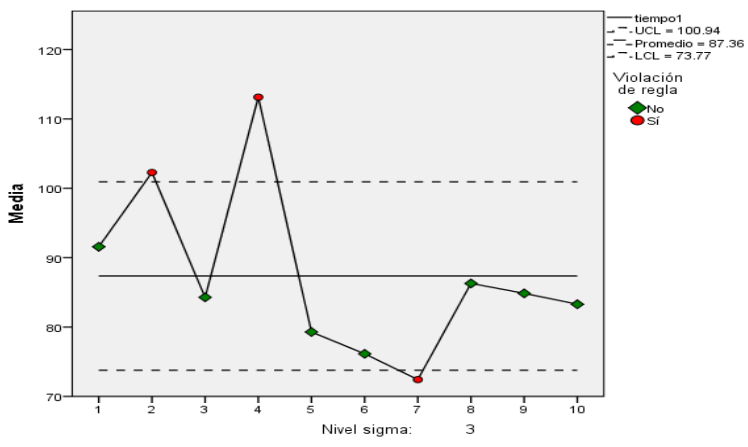

Figura 1. Promedio del tiempo de enfriamiento.

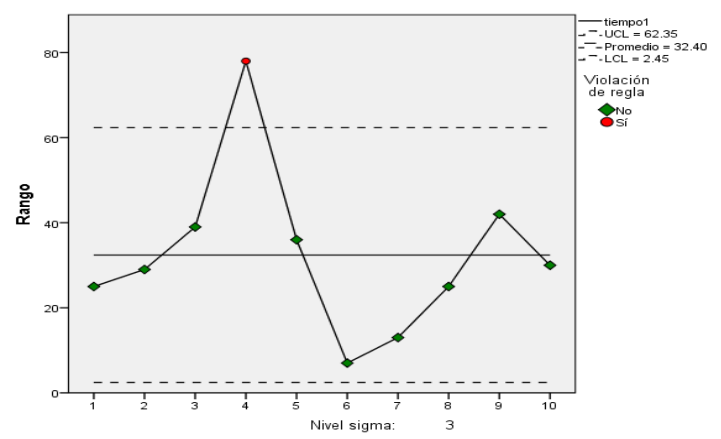

Figura 2. Rango del tiempo de enfriamiento.

El tiempo de enfriamiento es un factor importante que se ve influido por distintos factores, tales como:

- Temperatura del lugar donde se elabora el producto, ya que los estantes donde se colocan las bandejas están en el mismo cuarto donde están las pailas.

- Temperatura final de cocción, que sería la temperatura a la que se extrae el producto y se coloca en las bandejas para su enfriamiento; por lo que entre más caliente esté la mezcla más tardará en enfriarse.

- Cantidad de merma (producto sobrante) que se extrae de las bandejas, que no está dentro de la forma rectangular en la que se vende el producto, este es un factor importante, ya que al agregar la merma se baja la temperatura de la mezcla y se enfrían más rápido las bandejas.

- Posición en donde se coloca la bandeja en los estantes. Este factor se da porque el abanico que utilizan para acelerar un poco el proceso de enfriamiento solo tiene alcance a las bandejas de la parte superior de los estantes.

Por los factores anteriores la empresa tiene un tiempo promedio de enfriamiento de las bandejas de 100 minutos, lo que sería nuestra especificación superior, para poder hacer el análisis de capacidad, en donde se utilizaría el análisis de capacidad del proceso con una sola especificación.

$$
\mathrm{C}_{\mathrm{pk}}=\frac{\mathrm{ES}-\overline{\mathrm{x}}}{3 \mathrm{~S}}=\frac{100-87.80952}{3(10.66287)}=0.38
$$

Según el valor del índice de 0.38 calculado y redondeando el valor a 0.3 , utilizando la tabla 9.2 [5] se obtiene que el proceso está $18.40 \%$ fuera de una especificación, que sería el 
porcentaje que está fuera de la especificación superior (especificación utilizada para el análisis de los datos obtenidos).

Los valores sobre 1.25 se consideran como índices de capacidad satisfactorio, pero si el valor es menor que 1 no cumple con esta regla; diciendo así que con un Cpk de 0.38, resultado de nuestros cálculos, se puede notar que el proceso no tiene capacidad. Esto significa que el proceso no es capaz de producir de manera estable dentro de los límites de especificación.

3.2 Mejoras propuestas por el equipo de trabajo para disminuir el tiempo de enfriamiento de las bandejas de achiote

1. Estandarizar la cantidad de merma utilizada en las pailas, ya que la utilización de estas disminuye la temperatura de las bandejas.

2. Controlar mediciones de la cantidad de mezcla vertida en cada bandeja utilizando un envase cuyas medidas puedan ser visibles y de fácil lectura.

3. Cambiar la balanza o calibración de esta, para poder tener un control más preciso de la cantidad de ingredientes de la misma mezcla para así evitar desperdicios de materia prima y garantizar la misma composición de los productos obtenidos.

4. Establecer un mantenimiento semanal de las bandejas o moldes, para evitar que las mismas presenten inconvenientes como inclinaciones, que no embonen bien los dos componentes de la bandeja, entre otros. Esto ayuda a que la mezcla se solidifique en barra de la mejor forma.

5. Disminuir la temperatura de vertido, luego de agregada la cantidad estándar de merma a la mezcla, en un rango propuesto de entre $45-50^{\circ} \mathrm{C}$.

6. Propuesta de cambio de distribución de la habitación donde se realiza todo este proceso manual:

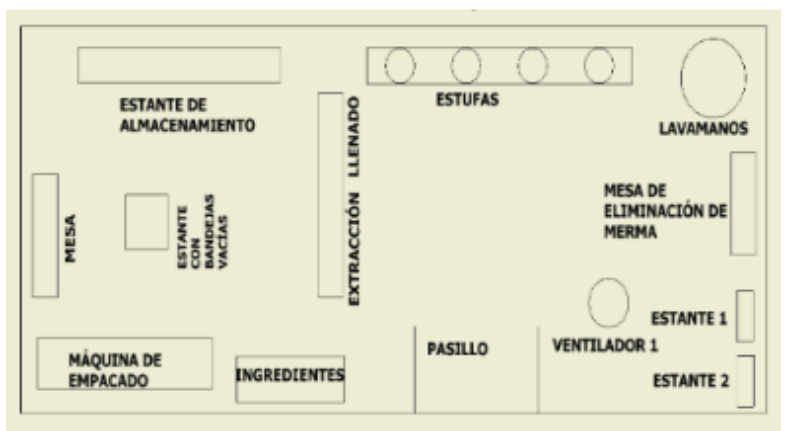

Figura 3. Distribución actual de la planta.

Recomendaciones en la modificación:

- La mesa debe evaluarse para observar si tiene objetos innecesarios que deben ser eliminados, si no es necesaria su presencia en el proceso, eliminarla. En el caso contrario se propone que su nueva ubicación sea vertical al lavamanos, debido a que donde está ubicada estorba al operario.
- El estante de almacenamiento es muy grande, observar si se necesita tanto espacio. Se sugiere que su nueva ubicación sea en donde se encuentran los estantes uno y dos, porque el lado izquierdo se considera más fresco y se necesitaba colocar los estantes uno y dos allá.

- Se propone que se mueva el ventilador uno (ver figura 4) y que se añada un segundo ventilador. El ángulo del ventilador no debe ser el actual, ya que con este ángulo solamente se refrescan las bandejas colocadas arriba del estante. El nuevo ángulo debe ser más obtuso de acuerdo con el soporte del techo. Además, si se puede colocar un ventilador de forma que gire, sería mejor.

- Para los estantes uno y dos se recomienda que se muevan al lugar donde se encuentra la mesa, porque hay más distancia de las estufas, y que se muevan con sus respectivos ventiladores.

- La mesa de eliminación de merma se propone que se mueva a donde se encuentra el estante de almacenamiento.

- El estante con bandejas vacías se puede colocar al lado de la nueva ubicación de los estantes uno y dos. Pero se recomienda que se evalúe si se puede utilizar para colocar nuevas bandejas o quitarlo si es innecesario.

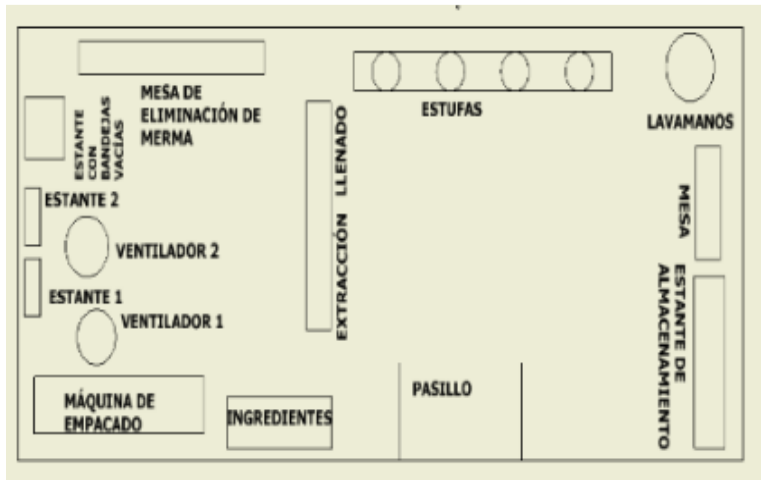

Figura 4. Distribución propuesta de la planta.

\subsection{Datos recolectados después de las mejoras}

Después de haberse implementado las mejoras uno y cinco; se recolectaron nuevos datos para demostrar si había una disminución en el tiempo de enfriamiento y de igual forma si se había conseguido el control del proceso. En la tabla 2 se presentan los nuevos datos obtenidos. Con estos datos se realizan los gráficos de control estadístico que se muestran en las figuras 5 y 6 .

Tabla 2. Datos recolectados del tiempo de enfriamiento de las bandejas de achiote condimentado en minutos

\begin{tabular}{|c|c|c|c|c|c|c|c|}
\hline \multicolumn{8}{|c|}{ Bandejas } \\
\hline SG. & 1 & 2 & 3 & 4 & 5 & 6 & 7 \\
\hline 1 & 81 & 81 & 83 & 90 & 90 & 96 & 96 \\
\hline 2 & 68 & 83 & 76 & 83 & 77 & 83 & 84 \\
\hline 3 & 84 & 78 & 77 & 81 & 83 & 96 & 99 \\
\hline 4 & 81 & 81 & 83 & 84 & 87 & 90 & 94 \\
\hline 5 & 68 & 70 & 73 & 75 & 76 & 74 & 84 \\
\hline
\end{tabular}




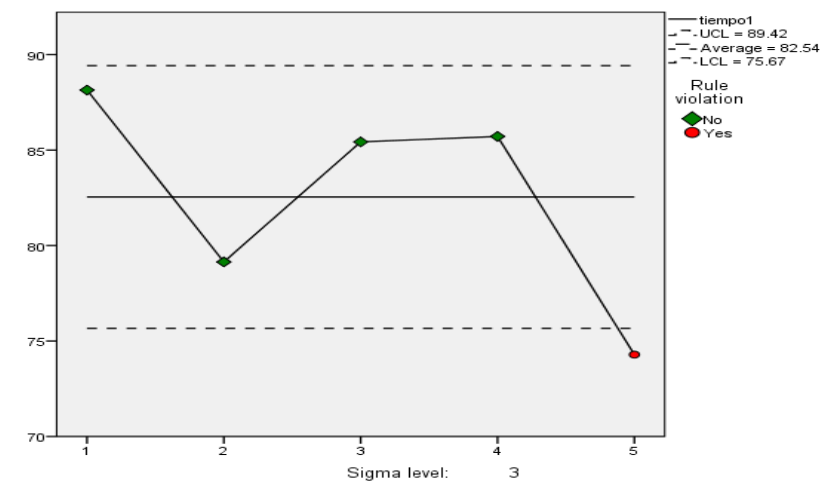

Figura 5. Promedio del tiempo de enfriamiento.

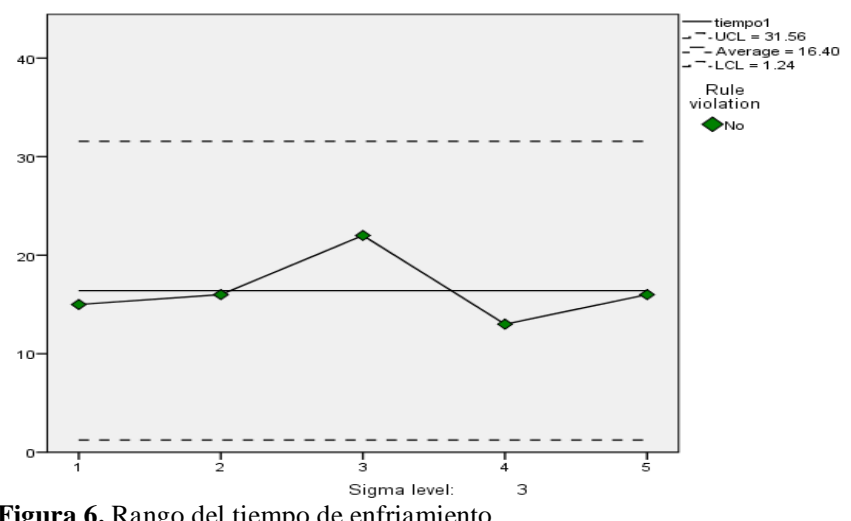

Figura 6. Rango del tiempo de enfriamiento.

Al calcular nuevamente la capacidad del proceso se obtiene:

$$
C_{p k}=\frac{E S-\bar{x}}{3 S}=\frac{100-82.54}{3(7.9014)}=0.736
$$

Según el valor del índice de 0.736 calculado y redondeando el valor a 0.7, utilizando la tabla 9.2 [5] en la sección con referencia a una sola especificación se obtuvo que el proceso posee un porcentaje fuera de una especificación de 1.7864, que sería el porcentaje que está fuera de la especificación superior.

Para aumentar la capacidad del proceso, se debe disminuir la desviación estándar de los datos, y en este caso ir disminuyendo el promedio del tiempo de enfriamiento.

Realizando el gráfico 1 donde se puede comparar en una gráfica de promedio de tiempo de enfriamiento de las bandejas de achiote, todos los datos obtenidos manteniendo los límites del grafico antes de las mejoras, se observa una disminución notable en el tiempo de enfriamiento cuando se aplican las mejoras que son los últimos cinco datos obtenidos.

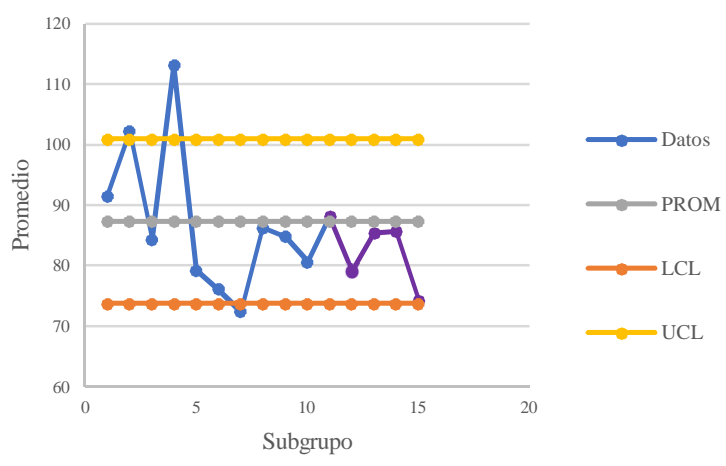

Grafico 1. Promedio de tiempo de enfriamiento de las bandejas de achiote.

\section{Análisis}

Con las dos propuestas implementadas se observa una mejora notable en el tiempo de enfriamiento, y se consigue la disminución de este tiempo, logrando a su vez que el proceso estuviese en control con un punto fuera del límite inferior, pero este punto es una causa asignable positiva que merece ser investigada para conocer lo que ocurrió. Además, se aumenta la capacidad, casi se duplicó la capacidad del proceso, pero es necesario seguir implementando otras mejoras para lograr que el proceso esté en capacidad.

De esta manera, las propuestas de la cantidad de merma vertida en las pailas y la disminución en la temperatura al verter en las bandejas dieron como resultado un mejor proceso en general; el tiempo de enfriamiento disminuyó lo que hace que el proceso tome menos tiempo, de tal manera que se pueda obtener un aumento en la productividad del proceso.

La investigación sirvió de base para demostrar que al aplicar las métricas de Seis Sigma en un proceso real se consigue mejorar los índices de capacidad, así como el índice Seis Sigma del proceso, y que si se implementan más mejoras se logrará una notable disminución del tiempo de enfriamiento, lo que daría como resultado una mejor fabricación del achiote condimentado lo que a su vez, produciría mayores ganancias a la empresa.

\section{Conclusiones y recomendaciones}

- Con solo implementar dos de las mejoras propuestas se observó que hubo cambios en el tiempo de enfriamiento, el cual disminuyó.

- Se estudió el proceso por medio de control charts, el cual permitió controlar ciertas mediciones que estuvieron por encima de sus respectivos límites. Al no contar con mucho tiempo no se pudo obtener muchos datos y llevar a cabo los control charts por un periodo de tiempo más prolongado.

- La posición de cómo se colocaron las bandejas influyó, y el que el ventilador estuviera más cerca también, ambas favorables para el tiempo de enfriamiento.

- Implementando las dos mejoras se observó que la capacidad aumentó debido a que se notó cambios en el 
tiempo de enfriamiento, así como también, se aumentó la productividad del proceso gracias a la disminución del tiempo de enfriamiento de proceso.

- Se recomienda implementar más mejoras para poder obtener un tiempo de enfriamiento menor al que se obtuvo.

\section{AGRADECIMIENTOS}

A Dios por darnos la fuerza, salud y sabiduría, durante el tiempo de aplicación y finalización del proyecto.

A la señora Romelia Marín y a su hija la Ingeniera Keiko Onodera, por abrirnos las puertas de su prestigiosa empresa, a la Lcda. Lourdes Batista y al Ing. Cristóbal Sierra y a todo el equipo de colaboradores, encargados del proceso de producción del achiote condimentado, por su cooperación y disponibilidad durante todo el proceso.

\section{REFERENCIAS}

[1] R. A. y. A. SATURNO, «Alternativa para los productores de achiote, máquina desgranadora manual.,» Revista UTP, vol. I, ${ }^{\circ}$ 1, p. 46, 2002.

[2] V. autores, «Milenio,» Grupo Milenio, 27 Octubre 2016. [En línea]. Available: http://www.milenio.com/opinion/variosautores/universidad-politecnica-de-tulancingo/control estadistico-de-la-calidad. [Último acceso: 14 Junio 2018]

[3] R. S. R. y. B. W. T. III, Operations Management, Quality and Competitiveness in a Global Environment, United States of America: John Wiley \& Sons, Inc., 2006.

[4] H. G. P. y. R. d. 1. V. Salazar, Control estadístico de la calidad y Seis Sigma, México: McGraw Hill, 2013.

[5] H. G. Pulido, «Índices de capacidad de procesos,» de Calidad total y productividad, México, McGraw Hill, 2010, p. 168. 